\title{
Entropic multiple-relaxation-time multirange pseudopotential lattice Boltzmann model for two-phase flow
}

\author{
Feifei Qin, ${ }^{1,2, a)}$ Ali Mazloomi Moqaddam, ${ }^{2}$ Qinjun Kang, ${ }^{3}$ Dominique Derome, ${ }^{2}$ \\ and Jan Carmeliet ${ }^{1,2}$ \\ ${ }^{1}$ Chair of Building Physics, Department of Mechanical and Process Engineering, ETH Zürich \\ (Swiss Federal Institute of Technology in Zürich), Zürich 8093, Switzerland \\ ${ }^{2}$ Laboratory of Multiscale Studies in Building Physics, Empa (Swiss Federal Laboratories for Materials Science \\ and Technology), Dübendorf 8600, Switzerland \\ ${ }^{3}$ Earth and Environment Sciences Division (EES-16), Los Alamos National Laboratory (LANL), Los Alamos, \\ New Mexico 87545, USA
}

(Received 22 November 2017; accepted 25 February 2018; published online 22 March 2018)

\begin{abstract}
An entropic multiple-relaxation-time lattice Boltzmann approach is coupled to a multirange ShanChen pseudopotential model to study the two-phase flow. Compared with previous multiplerelaxation-time multiphase models, this model is stable and accurate for the simulation of a two-phase flow in a much wider range of viscosity and surface tension at a high liquid-vapor density ratio. A stationary droplet surrounded by equilibrium vapor is first simulated to validate this model using the coexistence curve and Laplace's law. Then, two series of droplet impact behavior, on a liquid film and a flat surface, are simulated in comparison with theoretical or experimental results. Droplet impact on a liquid film is simulated for different Reynolds numbers at high Weber numbers. With the increase of the Sommerfeld parameter, onset of splashing is observed and multiple secondary droplets occur. The droplet spreading ratio agrees well with the square root of time law and is found to be independent of Reynolds number. Moreover, shapes of simulated droplets impacting hydrophilic and superhydrophobic flat surfaces show good agreement with experimental observations through the entire dynamic process. The maximum spreading ratio of a droplet impacting the superhydrophobic flat surface is studied for a large range of Weber numbers. Results show that the rescaled maximum spreading ratios are in good agreement with a universal scaling law. This series of simulations demonstrates that the proposed model accurately captures the complex fluid-fluid and fluid-solid interfacial physical processes for a wide range of Reynolds and Weber numbers at high density ratios. Published by AIP Publishing. https://doi.org/10.1063/1.5016965
\end{abstract}

\section{INTRODUCTION}

Multiphase flows are ubiquitously seen across different fields. Examples from nature are droplets' formation in clouds or raindrop impact on surfaces. Other examples can be found in engineering applications, such as spray coating and cooling, ${ }^{1}$ ink-jet printing, ${ }^{2}$ fuel droplets' impact on the wall of a combustion chamber, etc. Over the last decades, lattice Boltzmann (LB) models have been developed to be powerful tools for simulating a multiphase flow. ${ }^{3-5}$ Situated at the mesoscopic scale between micro and continuum, LB models solve a fully discrete kinetic equation for particle distribution functions. Due to their kinetic nature, LB models can capture the interfacial dynamics automatically by incorporating intermolecular-level interactions, a feature which is very advantageous over traditional computational fluid dynamics (CFD) methods such as the volume of fluid $(\mathrm{VOF})^{6,7}$ or level-set method ${ }^{8,9}$ that require interface tracking and reconstruction.

In general, there are four main categories of multiphase LB models: the Shan-Chen pseudopotential model, ${ }^{10,11}$ free energy model, ${ }^{12}$ color-gradient model, ${ }^{13}$ and mean-field

\footnotetext{
a) Author to whom correspondence should be addressed: fqin@ethz.ch and feifei.qin@empa.ch
}

model. ${ }^{14,15}$ Due to its simplicity and versatility, the Shan-Chen pseudopotential model is very popular among LB users and developers. In this model, the intermolecular interactions are represented with a density-dependent pseudopotential called effective mass, and the phase separation is achieved by imposing a nearest-neighbor attraction between different phases. Since its introduction, the pseudopotential model has been widely used in multiphase applications. ${ }^{16,17}$ However, the original model has some disadvantages, such as large spurious currents around interfaces at a large density ratio. It has been found that the spurious currents can be reduced by using high-order isotropic gradient operators ${ }^{18}$ or by increasing the interface thickness. ${ }^{19}$ Another disadvantage is that the surface tension is not tunable independent of the density or temperature ratio. To overcome these disadvantages, a multirange potential is proposed in Ref. 19, in which the interaction involves not only the nearest-neighbor lattices but also the next-nearestneighbor lattices. By introducing multirange interactions, it becomes possible to choose the surface tension independent of the equation of state (EoS).

Further, a severe limitation in the application of multiphase models is their incapacity to achieve low fluid viscosity. As an example, the lattice-Bhatnagar-Gross-Krook (LBGK) model, ${ }^{20,21}$ which is the most popular LB model due to its 
simplicity and high computation efficiency, becomes unstable when a low kinematic viscosity is used. Since most pseudopotential multiphase models are extensions derived from LBGK, they are found to be inadequate to simulate low viscous multiphase flows. One possible way of improvement is the use of multiple-relaxation-time (MRT) LB models. ${ }^{22}$ With different relaxation times for the higher order moments, the simulation becomes more stable for low viscosity flows. A drawback of MRT is that the relaxation times for higher order moments have to be set by the users based on their experience. Moreover, it is still not easy for MRT-based multiphase models to achieve good results simultaneously for a large density ratio and low viscosity in dynamic cases. ${ }^{23,24}$ Another approach is called the entropic LB model (ELBM), ${ }^{25-27}$ in which entropy is introduced at each lattice and increased for each time step, making it possible to reach low viscosity values. The ELBM-based free energy multiphase model can successfully capture droplet dynamics. ${ }^{28-30}$ However, this ELBM is computationally expensive since an implicit entropy equation has to be solved numerically at each lattice for each time step. Recently, a new LB model called the entropic multiplerelaxation-time LB model (EMRT LBM) ${ }^{31}$ proposed and applied for a turbulent flow, can span a wide range of fluid viscosity. EMRT LBM, an advanced MRT LBM, maximizes the entropy at each lattice for each time step by relaxing higher order moments properly. Moreover, the relaxation parameter is not user defined as in MRT LBM but can be obtained from analytically solving the entropy-maximum equation. As a result, there is no need to solve the implicit entropy equation like in ELBM, which results in important savings of computation time.

In this paper, an entropic multiple-relaxation-time multirange pseudopotential lattice Boltzmann model (EMRT-MP LBM) is proposed. With this new method, low fluid viscosity can be reached at a large density ratio and the surface tension is tunable independent of the density ratio. This method allows the study of different droplet dynamics over a wide range of Reynolds and Weber numbers at a high density ratio. This paper is divided into two main parts. In the first part, the theory of EMRT-MP LBM is presented. In the second part, results and discussions for a droplet splashing on a liquid film and for a droplet impacting a flat surface are studied and compared with empirical equations and experimental results in order to demonstrate the capabilities of the proposed LB implementation.

\section{NUMERICAL MODEL}

\section{A. EMRT LBM}

\section{General LBM}

A general form of the LB equation can be written as

$$
f_{i}\left(\mathbf{x}+\mathbf{v}_{i}, t+1\right)=f_{i}^{\prime} \equiv(1-\beta) f_{i}(\mathbf{x}, t)+\beta f_{i}^{\text {mirr }}(\mathbf{x}, t),
$$

where $f_{i}(\mathbf{x}, t)$ is the density distribution function at $\mathbf{x}$ lattice, $\mathrm{t}$ is the time, and $\mathbf{v}_{i}, i=1, \ldots, Q$ are the velocity vectors. The left-hand side is the propagation term, and the right-hand side $f_{i}^{\prime}$ represents the post-collision process. The mirror state $f_{i}^{\text {mirr }}$ is vital, affecting the performance of LB schemes. In the frequently used LBGK model, ${ }^{20,21}$ the mirror state is written as

$$
f_{i}^{m i r r}=2 f_{i}^{e q}-f_{i}
$$

in which $f_{i}^{e q}$ is the equilibrium density distribution function to maximize the entropy

$$
S[f]=-\sum_{i=1}^{Q} f_{i} \ln \left(\frac{f_{i}}{W_{i}}\right),
$$

where the weights $W_{i}$ are lattice-specific constants. To recover the Navier-Stokes equation, the kinematic viscosity of fluid is expressed as

$$
v=c_{s}^{2}\left(\frac{1}{2 \beta}-\frac{1}{2}\right),
$$

where $\beta$ is a free parameter related to kinematic viscosity and $c_{s}$ is the speed of sound that equals to $1 / \sqrt{3}$ (lattice unit). The macroscopic variables, i.e., density and velocity, can be obtained from

$$
\rho=\sum_{i=1}^{Q} f_{i}^{e q}
$$

and

$$
\rho \mathbf{u}=\sum_{i=1}^{Q} \mathbf{v}_{i} f_{i}^{e q} .
$$

The equilibrium distribution $f_{i}^{e q}$ used in this paper is the product form as proposed in Refs. 31 and 32 and is found in the supplementary material.

\section{Mirror state in EMRT LBM}

As explained above, $f_{i}^{\text {mirr }}$ in Eq. (1) is the key to proper performance of the LB scheme. We now present how it is constructed in EMRT LBM. ${ }^{31,32}$

The population of the density distribution function is basically split into three parts,

$$
f_{i}=k_{i}+s_{i}+h_{i},
$$

where $k_{i}$ is the kinematic part which depends on the locally conserved fields, $s_{i}$ is the shear part, which involves the stress tensor, and $h_{i}$ is the higher-order moment, which is a linear combination of the remaining high order moments that are not included in $s_{i}$. The mirror state can then be written in a one-parameter form as

$$
f_{i}^{m i r r}=k_{i}+\left[2 s_{i}^{e q}-s_{i}\right]+\left[(1-\gamma) h_{i}+\gamma h_{i}^{e q}\right],
$$

in which $\gamma$ is a parameter discussed below and the terms $s_{i}^{e q}$ and $h_{i}^{e q}$ denote $s_{i}$ and $h_{i}$ evaluated at equilibrium, respectively. The kinematic part $k_{i}$ is a fixed formula according to the conservation of mass and momentum. In Ref. 32, several formulations of the shear part $s_{i}$ are introduced. Here we apply the "KBC$\mathrm{N} 4$ " formulation. After defining the shear moments, the main problem lies in how to specify the value of $\gamma$. For any chosen $\gamma$, the resulting LB model still recovers the same kinematic viscosity $v$ since only higher order moments are modified. For example, $\gamma=2$ and $\gamma=1 / \beta$ result in the LBGK and regularized LB models, respectively.

In EMRT LBM, the value of $\gamma$ is calculated based on maximum of entropy $S\left[f^{\prime}(\gamma)\right]$ of the post-collision state $f^{\prime}$. 
With introducing $\Delta s_{i}=s_{i}-s_{i}^{e q}$ and $\Delta h_{i}=h_{i}-h_{i}^{e q}$, the condition for the critical point, i.e., where the derivative of entropy equals zero, reads as ${ }^{32}$

$$
\sum_{i=1}^{Q} \Delta h_{i} \ln \left[1+\frac{(1-\beta \gamma) \Delta h_{i}-(2 \beta-1) \Delta s_{i}}{f_{i}^{e q}}\right]=0 .
$$
as $^{32}$

The approximated explicit solution for $\gamma$ can be written

$$
\gamma=\frac{1}{\beta}-\left(2-\frac{1}{\beta}\right) \frac{\langle\Delta s, \Delta h\rangle}{\langle\Delta h, \Delta h\rangle},
$$

where the entropic scalar product $\langle X, Y\rangle$ in the $Q$ dimensional space is

$$
\langle X, Y\rangle=\sum_{i=1}^{Q} \frac{X_{i} Y_{i}}{f_{i}^{e q}} .
$$

With the main points described above, we organize the whole procedure of implementing the EMRT LB scheme as follows: ${ }^{32}$

1. compute conserved quantities $\rho, \mathbf{u}$,

2. compute equilibrium $f_{i}^{e q}(\rho, \mathbf{u})$,

3. compute shear part $s$ and $s^{e q}$,

4. compute $\Delta s_{i}=s_{i}-s_{i}^{e q}$,

5. compute $\Delta h_{i}=h_{i}-h_{i}^{e q}=f_{i}-f_{i}^{e q}-\Delta s_{i}$,

6. compute the value of $\gamma$,

7. $\operatorname{relax} f_{i}^{\prime}=f_{i}-\beta\left(2 \Delta s_{i}+\gamma \Delta h_{i}\right)$.

\section{B. Pseudopotential model}

\section{Fluid-fluid interaction}

At given initial conditions, a fluid system undergoes segregation into different phases as a result of the forces between the molecules. In Shan and Chen's pseudopotential model, the fluid-fluid interaction force is modeled as ${ }^{10,11}$

$$
\mathbf{F}_{c}=-G \psi(\mathbf{x}) \sum_{i=1}^{Q} w\left(\left|\mathbf{v}_{i}\right|^{2}\right) \psi\left(\mathbf{x}+\mathbf{v}_{i}\right) \mathbf{v}_{i},
$$

in which $\psi$ is the interaction potential or the so-called effective mass, $G$ is the interaction strength, and $w\left(\left|\mathbf{v}_{i}\right|^{2}\right)$ are the weights. For D2Q9 lattices, the weights are $w(1)=1 / 3$ and $w(2)=1 / 12$, while for D3Q27 lattices, they are $w(1)=1 / 6$, $w(2)=1 / 12$, and $w(3)=0$. The $w\left(\left|\mathbf{v}_{i}\right|^{2}\right)$ here and the $W_{i}$ of Eq. (3) are different parameters.

As proposed in Ref. 33, the equation of state $P_{E o S}$ is expressed as

$$
P_{E o S}=\rho c_{s}^{2}+G c^{2} \psi^{2} / 2
$$

where the interaction potential equals

$$
\psi=\sqrt{2\left(P_{E o S}-\rho c_{s}^{2}\right) / G c^{2}} .
$$

To realize large density ratios, ${ }^{34}$ we use the Peng-Robinson (P-R) equation of state

$$
P_{E o S}=\frac{\rho R T}{1-b \rho}-\frac{a \alpha(T) \rho^{2}}{1+2 b \rho-b^{2} \rho^{2}}
$$

with

$$
\begin{aligned}
\alpha(T)= & {\left[1+\left(0.37464+1.5422 \omega-0.26992 \omega^{2}\right)\right.} \\
& \left.\times\left(1-\sqrt{T / T_{c}}\right)\right]^{2},
\end{aligned}
$$

in which the attraction and repulsion parameters are $a=0.45724 R^{2} T_{c}^{2} / p_{c}, \quad b=0.0778 R T / p_{c}$ and $T_{c}$ and $p_{c}$ represent critical temperature and pressure, respectively. $T$ is the temperature, and $R$ is the gas constant. In simulations, ${ }^{34}$ the parameters are usually set as $a=2 / 49, b=2 / 21$, and $R=1$. The acentric parameter $\omega$ in Eq. (16) depends on the liquid and $\omega=0.344$ for water. With these values, the liquid-vapor interface is around 4-5 lattices thick, but spurious currents are still very large especially at low viscosity and high density ratios. Following the idea that the interface thickness is proportional to $1 / \sqrt{a}{ }^{35}$ we set $a=1 / 98, b=2 / 21$, and $R=1$ to reduce spurious currents around interface, while ensuring thermodynamic consistency. A drawback is that the interface thickness is relatively thick, around 6-7 lattices.

\section{Multirange potential}

Theoretically, the surface tension, i.e., the fluid-fluid interaction force per unit length, can be tuned by adjusting the interaction strength $G$. However, as the interaction potential relates to effective mass as $\psi \propto G^{-1 / 2}$ [Eq. (14)], the force becomes $\left|\mathbf{F}_{c}\right| \propto G \psi^{2} \propto 1$ [Eq. (12)]. This means that the surface tension cannot be tuned by changing $G$. To obtain a tunable interaction force, the multirange potential as proposed in Ref. 19 is applied,

$$
\mathbf{F}_{c}=-\psi(\mathbf{x}) \sum_{i=1}^{Q} w\left(\left|\mathbf{v}_{i}\right|^{2}\right)\left[G_{1} \psi\left(\mathbf{x}+\mathbf{v}_{i}\right)+G_{2} \psi\left(\mathbf{x}+2 \mathbf{v}_{i}\right)\right] \mathbf{v}_{i},
$$

while the equation of state is modified as

$$
P_{E o S}=\rho c_{s}^{2}+\left(G_{1}+2 G_{2}\right) c^{2} \psi^{2} / 2 .
$$

In this way, the surface tension can be tuned by varying the ratio $\left(G_{1}+8 G_{2}\right) /\left(G_{1}+2 G_{2}\right) \cdot{ }^{19}$ It is worth mentioning that this tuning of the surface tension may also change the density ratio to some degree. We found however the effect of such tuning on the density ratio to be moderate which makes this approach adequate if a specific density ratio is not required.

\section{Fluid-solid adhesion}

Several types of fluid-solid interaction forces are compared in Ref. 36. To realize a full range of contact angles, we use the following fluid-solid interaction force adapted for a multirange potential approach:

$$
\begin{aligned}
\mathbf{F}_{w}= & -\psi(\mathbf{x}) \sum_{i=1}^{Q} w\left(\left|\mathbf{v}_{i}\right|^{2}\right)\left[G_{1} \psi\left(\rho_{w}\right) I\left(\mathbf{x}+\mathbf{v}_{i}\right)\right. \\
& \left.+G_{2} \psi\left(\rho_{w}\right) I\left(\mathbf{x}+2 \mathbf{v}_{i}\right)\right] \mathbf{v}_{i},
\end{aligned}
$$

where $I$ is the indicator function that equals unity at solid nodes and zero at fluid nodes and $\rho_{w}$ is the parameter to determine wettability. With this fluid-solid interaction force, surface wettability can be defined as

$$
w=\left(G_{1}+G_{2}\right) \psi\left(\rho_{w}\right) .
$$

Denoting the wettability at contact angle $90^{\circ}$ as $w_{90}$, we can define the relative wettability as

$$
w_{r}=w-w_{90},
$$


which equals zero at a contact angle of $90^{\circ}$. The contact angle is approximately linearly dependent on (relative) wettability.

\section{Body force}

A constant body force such as gravity is expressed as

$$
\mathbf{F}_{g}=\rho \mathbf{g},
$$

where $\mathbf{g}$ is the acceleration due to body force.

\section{Force scheme}

To incorporate the forces mentioned above, we adopt the exact difference method (EDM) developed in Ref. 37, which is directly derived from the Boltzmann equation. In the EDM, a source term is added on the right-hand side of Eq. (1) as

$$
\begin{aligned}
f_{i}\left(\mathbf{x}+\mathbf{v}_{i}, t+1\right)= & f_{i}^{\prime} \equiv(1-\beta) f_{i}(\mathbf{x}, t)+\beta f_{i}^{m i r r}(\mathbf{x}, t) \\
& +f_{i}^{e q}(\rho, \mathbf{u}+\Delta \mathbf{u})-f_{i}^{e q}(\rho, \mathbf{u}),
\end{aligned}
$$

where $\mathbf{u}$ is obtained by Eq. (6) and $\Delta \mathbf{u}$ is expressed as

$$
\Delta \mathbf{u}=\frac{\mathbf{F} \delta t}{\rho},
$$

and $\mathbf{F}$ is the sum of all the forces as

$$
\mathbf{F}=\mathbf{F}_{c}+\mathbf{F}_{w}+\mathbf{F}_{g}
$$

\section{RESULTS AND DISCUSSION}

\section{A. Single stationary droplet}

To validate the EMRT-MP LBM, a single liquid droplet surrounded by vapor is first simulated in two dimensions. The domain size is $201 \times 201$ lattices $^{2}$ and the droplet with a radius of 50 lattices is located at the center of the domain.

The equilibrium state of the droplet for different temperature ratios is simulated, and the density of liquid and vapor versus temperature is plotted in Fig. 1. We can see from this figure that the simulation results follow Maxwell's equal area rule, which means our model is thermodynamically consistent for a large range of density or temperature ratios. Looking at

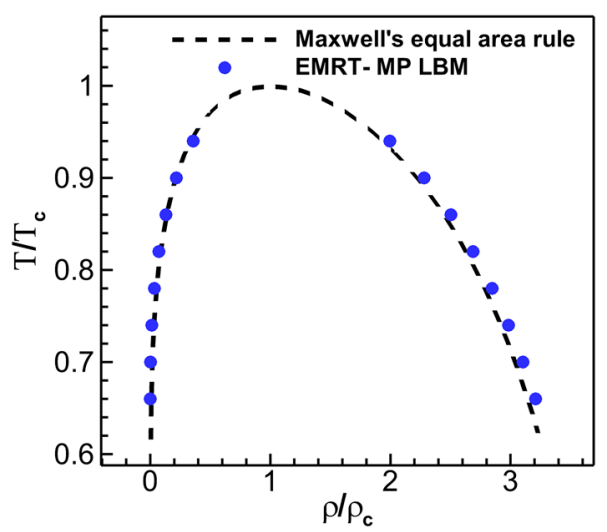

FIG. 1. Coexistence curve of liquid and vapor density for different temperatures. Circles: EMRT-MP LBM simulation; dashed line: Maxwell's equal area rule.

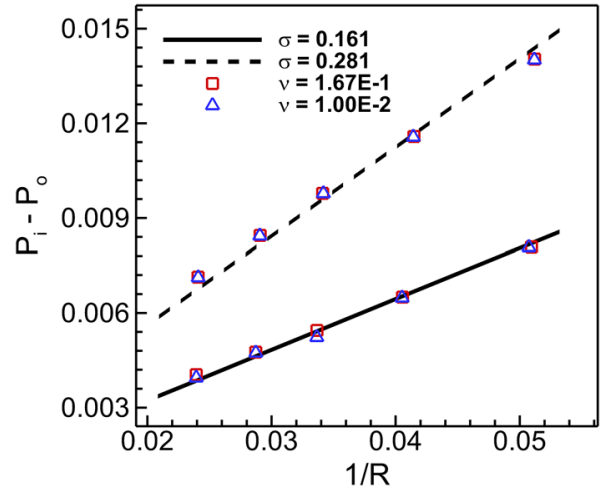

FIG. 2. Pressure difference versus the inverse of the radius of the droplet for two values of kinetic viscosity $(v)$ and surface tension $(\sigma)$. Symbols: EMRT-MP LBM simulation; lines: Laplace's law at two values of surface tension.

the pressure difference $\Delta p$ between the inner and outer regions of the droplet at equilibrium, we can see that the results comply with Laplace's law as

$$
\Delta p=\frac{\sigma}{R},
$$

where $\sigma$ is the surface tension and $R$ represents the droplet radius, as shown in Fig. 2. The droplet radius ranges from 20 to 40 lattices, and simulations are run with a temperature ratio $T / T_{c}=0.74$ and a density ratio $\rho_{l} / \rho_{v} \approx 223$. Different surface tensions can be obtained by varying the ratio $\left(G_{1}+8 G_{2}\right) /\left(G_{1}+2 G_{2}\right)$, and the simulations for two different viscosity values coincide as observed in Fig. 2.

Finally, we monitor the level of spurious current in our model. The maximum spurious current $\left|\mathbf{u}^{s}\right|_{\max }$ is defined as the maximum velocity magnitude of the entire domain. As shown in Fig. 3, $\left|\mathbf{u}^{s}\right|_{\max }$ increases with an increasing density ratio. Two values of kinematic viscosity are considered, and its effect is found not to be significant. Compared to the original pseudopotential model, the maximum spurious current is about one order of magnitude smaller in our model. To decrease the influence of spurious current while ensuring capturing the mechanism of two-phase flow, we always use a density ratio $\rho_{l} / \rho_{v} \approx 223$ at a temperature ratio $T / T_{c}=0.74$. The maximum

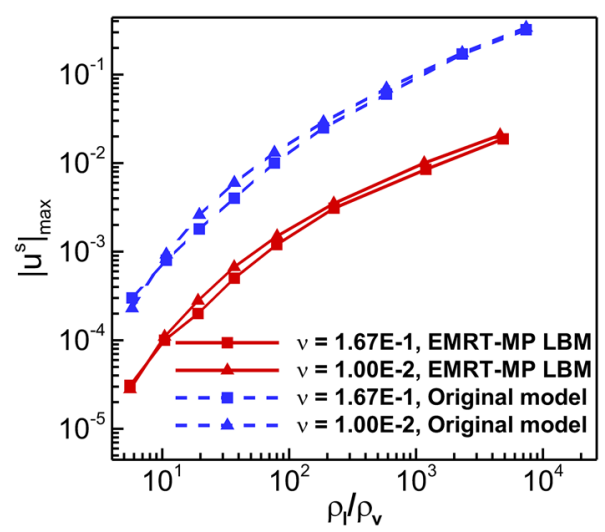

FIG. 3. Comparison of maximum spurious current around the liquid-vapor interface at various density ratios for two liquid kinematic viscosity values. Solid lines: EMRT-MP LBM; dashed lines: original pseudopotential model. 
spurious current is around $3.3 \times 10^{-3}$, which is sufficiently small to consider its influence negligible. Further, the relation of maximum spurious current versus the Ohnesorge number $\left(O h=\sqrt{W e} / \operatorname{Re}\right.$, with $\operatorname{Re}=U D_{0} / v_{l}$ and $\left.W e=\rho_{l} U^{2} D_{0} / \sigma\right)$ at $\rho_{l} / \rho_{v} \approx 223$ shows that with the Oh ranging from $3.0 \times 10^{-3}$ to 1.0 , the spurious current only slightly decreases from $3.3 \times 10^{-3}$ to $2.5 \times 10^{-3}$, which is almost independent of Oh. Finally, we verify that the maximum spurious current remains quasi-constant for three different grid sizes $N_{D}=100$, 150,200 of droplet diameter, which means the simulation is grid independent.

In conclusion, these validation and verification exercises show this model to be adequate to simulate a two-phase flow in static cases for different fluid viscosity and surface tension values at a high density ratio.

\section{B. Droplet splashing on liquid film}

Droplet splashing on a liquid film or solid surface has been studied in experiments. ${ }^{38-42}$ The study in Ref. 38 shows that the critical Weber number for droplet splashing on a very thin liquid film is insensitive to the film thickness while being dependent on fluid viscosity and surface characteristics. Experiments in Ref. 40 focus on providing a splash and non-splash boundary for droplet impact on a solid surface and liquid film with a critical capillary and Weber number. In Ref. 41, a droplet splash on a thin film is investigated with time on crown radius, height, film thickness, etc. Despite the experimental studies above, the general conditions for splashing are still not clear. In numerical studies, CFD methods ${ }^{43-46}$ are commonly used to study splashing, while LBM-based studies are found still incapable of reaching sufficiently high Reynolds and Weber numbers. $^{24,35,47}$

First, the conditions for droplet splashing when impacting on a liquid film are studied with EMRT-MP LBM. The size of the two-dimensional computational domain is 2400 $\times 1200$ lattices $^{2}$ with an initial liquid film thickness of $h=100$ lattices. The liquid sheet generated by droplet impact is very thin, and the secondary droplets are very small, which is why the high resolution grid is needed to capture these two aspects correctly. Due to this high resolution, it is now computationally not feasible for us to do a three-dimensional simulation. Therefore, we only do two-dimensional simulations in this section. The liquid and vapor densities are $\rho_{l}=7.82$ and $\rho_{v}=0.035$, respectively, at a temperature ratio of $T / T_{c}=0.74$. The initial droplet diameter is $D_{0}=200$ lattices, and the impact velocity is $\left(u_{x}, u_{y}\right)=(0,-U)$, where $U=0.15$ (lattice unit). For the boundary conditions, the left and right sides of the domain are considered periodic, while the top and bottom surfaces are considered as non-slip walls. We did not simulate half of the domain with a symmetry boundary condition because the flow at the symmetry boundary is very complex after the impact, leading to numerical divergence.

The Reynolds number, as the ratio of inertia and viscous forces, and the Weber number, as the ratio of kinematic and surface energies, are defined as $\operatorname{Re}=U D_{0} / v_{l}$ and $W e=\rho_{l} U^{2} D_{0} / \sigma$, respectively. In our simulations, the Weber number equals 220 and the Reynolds number is set successively to 100,1000 , and 5000 . The non-dimensional time is defined as $t^{*}=U t / D_{0}$. Snapshots during impingement for $R e=100,1000,5000$ are shown in Figs. 4-6 (Multimedia view), respectively.

At $R e=100$, a thin liquid sheet is emitted after droplet impact and then grows into a crown propagating radially away. The liquid sheet does not result in a splashing behavior, as shown in Fig. 4 (Multimedia view).

Increasing the Reynolds number to 1000, the liquid sheet forming the crown becomes thinner and thinner with time. Finally, the end rim of the sheet becomes unstable and breaks up into secondary small droplets. This splashing initiation can be seen clearly in Fig. 5 (Multimedia view) from $t^{*}=3.75$.

The simulations at $\mathrm{Re}=100$ and 1000 are run with both LBGK and EMRT-MP to compare the computation time. In running 10000 iterations with 16 processors, the time

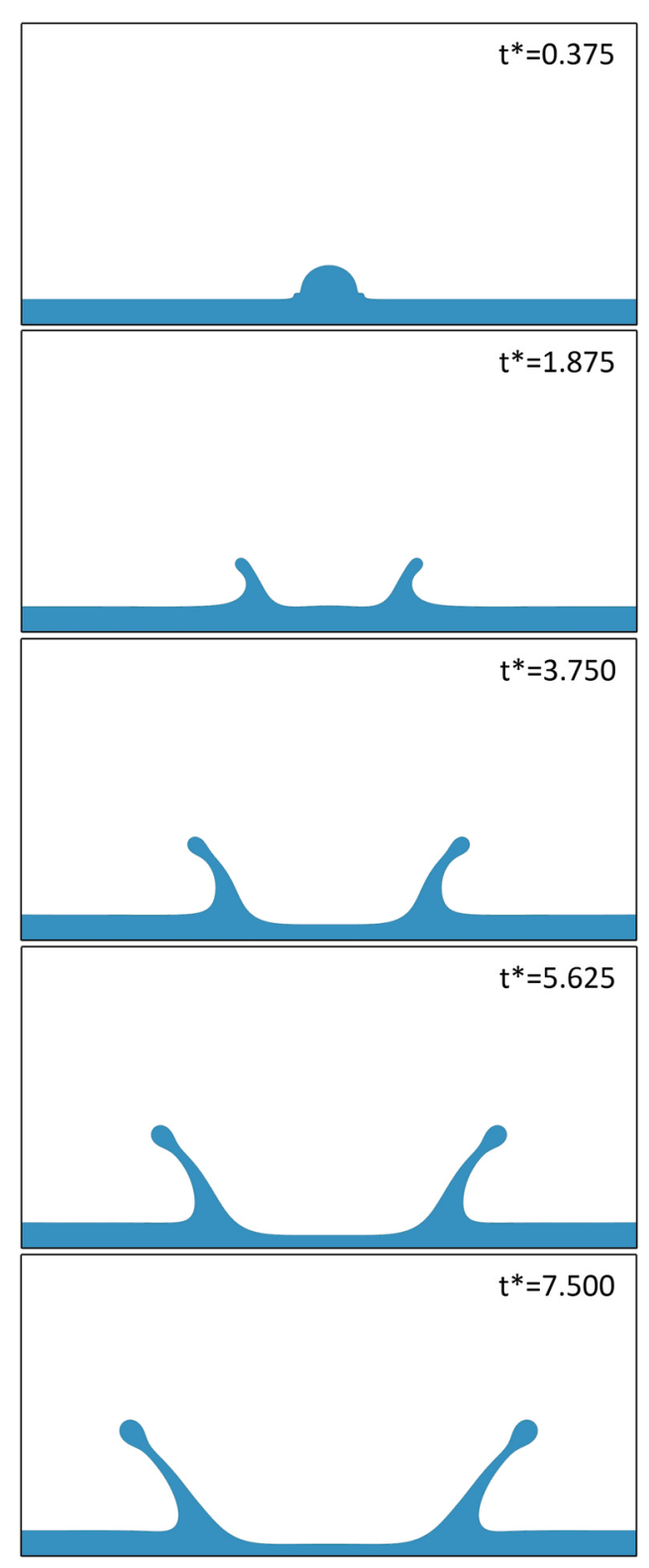

FIG. 4. Snapshots during droplet impingement at $\mathrm{Re}=100$ and We $=220$, with the liquid to vapor density ratio around 223. No splashing occurs. Multimedia view: https://doi.org/10.1063/1.5016965.1 


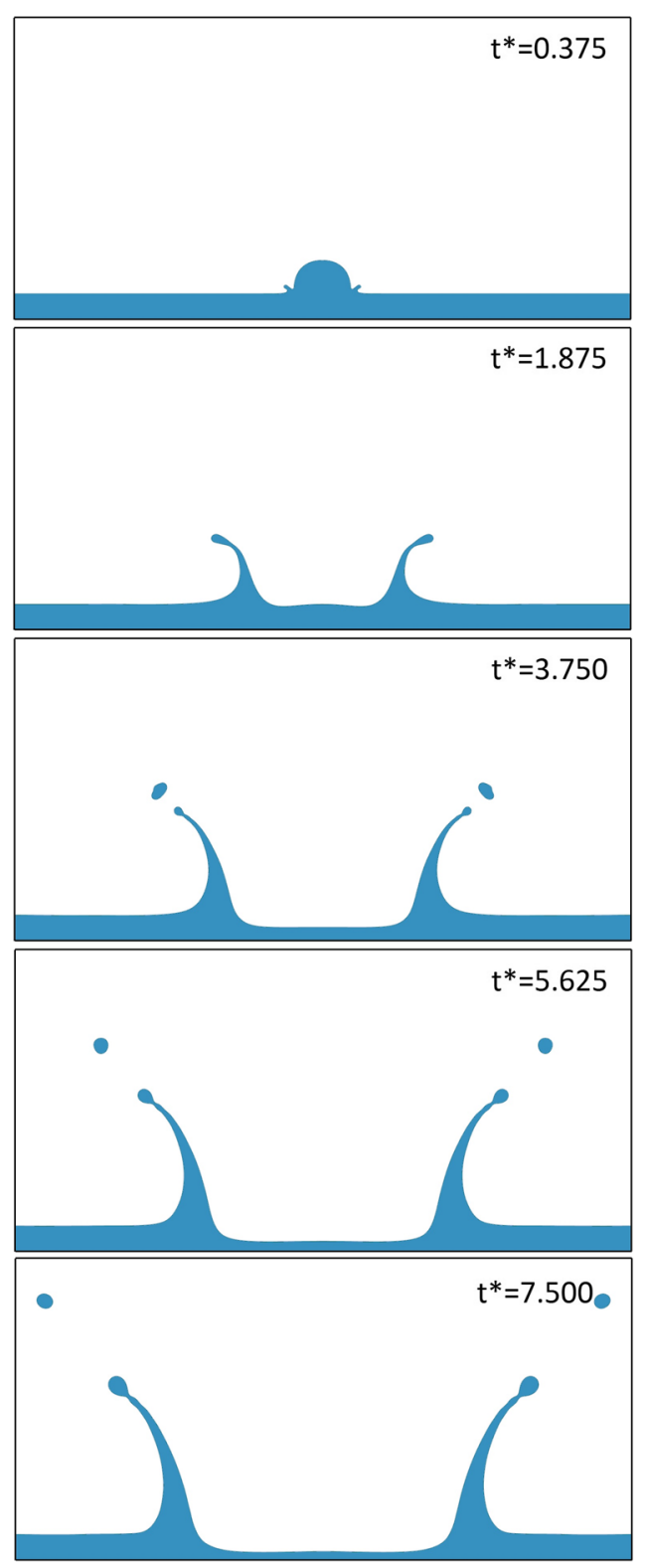

FIG. 5. Snapshots during droplet impingement at $\operatorname{Re}=1000$ and $\mathrm{We}=220$, with the liquid to vapor density ratio around 223. Splashing occurs. Multimedia view: https://doi.org/10.1063/1.5016965.2

consumed with LBGK and EMRT-MP is 120 and $134 \mathrm{~min}$, respectively. Our model thus costs $11.7 \%$ extra computation time, which is similar to previous MRT schemes reported to require $15 \%$ more time than LBGK in a single phase flow simulation. ${ }^{48}$ We remark that the case of $\mathrm{Re}=5000$ cannot anymore be handled by LBGK for the same setup parameters.

With EMRT-MP LBM, at a Reynolds number of 5000, the crown sheet formed does not break up immediately but becomes elongated with time showing a thinning process. When the sheet is sufficiently thin, it breaks into multiple secondary droplets, as shown in Fig. 6 (Multimedia view).

In Ref. 39, a parameter, called the Sommerfeld parameter, was introduced to predict the occurrence of splashing of a

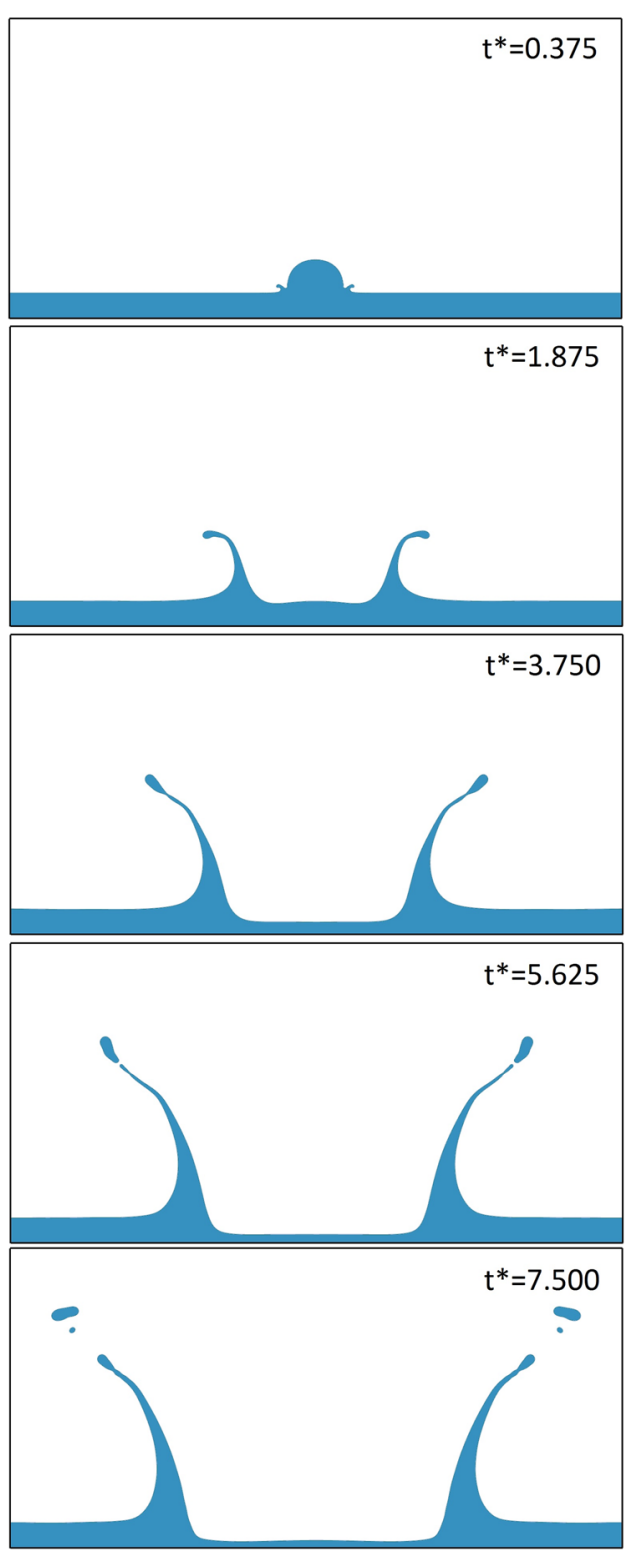

FIG. 6. Snapshots during droplet impingement at $\mathrm{Re}=5000$ and $\mathrm{We}=220$, with the liquid to vapor density ratio around 223. Splashing occurs with multiple secondary droplets. Multimedia view: https://doi.org/ $10.1063 / 1.5016965 .3$

droplet impacting liquid films or surfaces. It is defined as

$$
K=W e^{1 / 2} \operatorname{Re}^{1 / 4} .
$$

When $K$ is larger than the critical value $K_{c}$, splashing occurs.

An estimate of $K_{c}$ is proposed as $K_{c} \approx 57.7$ in Ref. 39. In Ref. 45 , Sommerfeld's law is recovered theoretically, and $K_{c}$ is found to be around 225 in axisymmetric CFD simulations. In our two-dimensional simulations, the Sommerfeld parameter is $\mathrm{K}=46.9,83.4,124.7$ for Reynolds numbers $\mathrm{Re}=100,1000$, 5000 , respectively. As our simulation is in two-dimension, we cannot use $K_{c} \approx 57.7$ or $K_{c} \approx 225$ as the critical value for the splash/non-splash boundary directly. However, our simulation results follow the qualitative trend that splashing occurs and becomes more intensive with the increase of the Sommerfeld parameter. 
It is worth to mention that, although our simulation is in two-dimension, the mechanism for splashing is the same as that of a real three-dimensional droplet: with an increasing inertial force, the viscous force and capillary force cannot hold the droplet as a whole anymore, and the droplet starts breaking into secondary droplets. A two-dimensional droplet splashing simulation at $\mathrm{Re}=1000$ and $\mathrm{We}=8000$ as done in Ref. 46 with the VOF showed that the splashing phenomenon observed is similar to our simulation. Further, we want to emphasize that the splashing is not due to numerical under-resolution. From Fig. 7, we can clearly see that the mesh is fine enough to eliminate the effect of liquid-vapor interface and to fully resolve the liquid sheet before and after splashing. Therefore, the generation of the secondary droplet is due to the large inertial force (compared with viscous and capillary force), and not due to numerical under-resolution.

We now consider the post-impact behavior. As theoretically explained in Ref. 45, after droplet impact, the spreading radius $r$ follows a square root of time law

$$
r / D_{0} \approx C \sqrt{U t / D_{0}}
$$

where the coefficient $C \approx 1.1$ for three-dimensional or axisymmetric modeling. ${ }^{45,46}$ For two-dimensional modeling, the
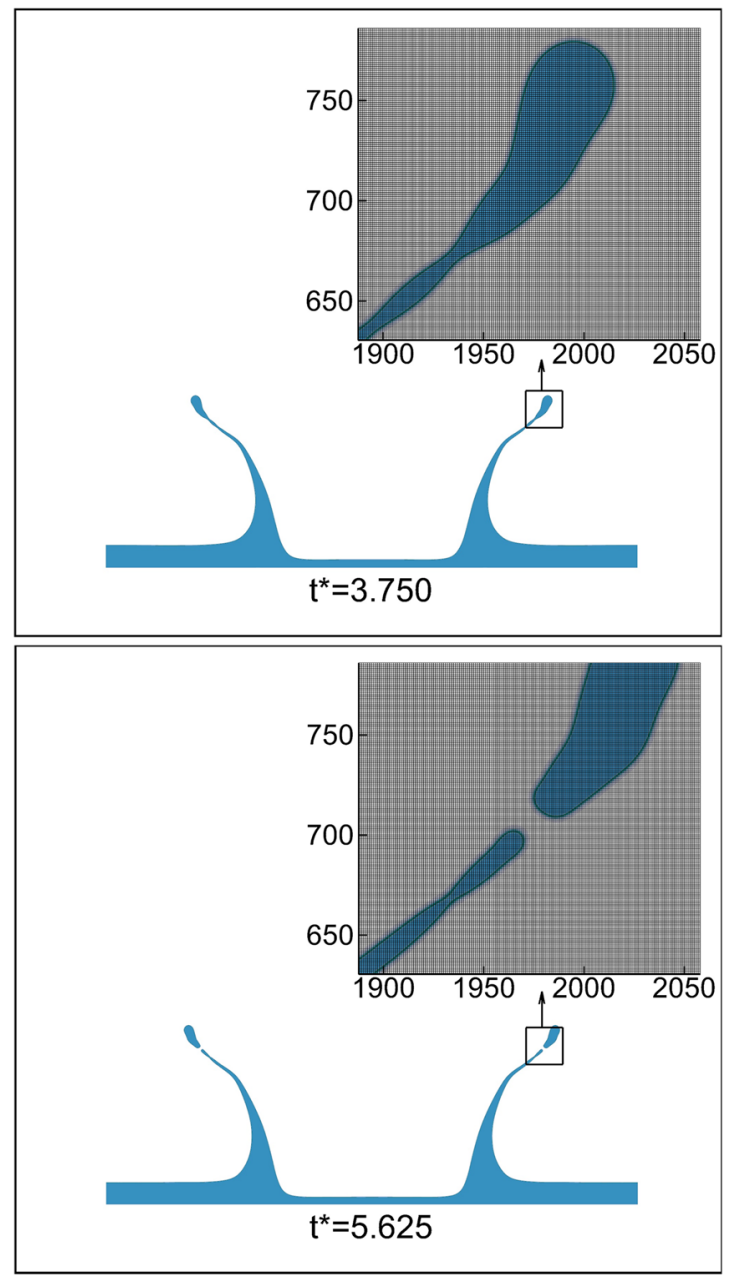

FIG. 7. Liquid sheet resolution before $\left(t^{*}=3.750\right)$ and after $\left(t^{*}=5.625\right)$ splashing at $\mathrm{Re}=5000$ and $\mathrm{We}=220$. coefficient is found to be larger than 1.1 in previous studies. ${ }^{24,35,47}$ The radius $r$ is defined at the intersection between the crown and the upper boundary of the liquid layer. ${ }^{45} \mathrm{In}$ Fig. 8, the spreading factor $r / D_{0}$ for different Reynolds numbers is plotted as a function of non-dimensional time $U t / D_{0}$. It is clear that the spreading radius is independent of the Reynolds number and the simulation results are in agreement with the square root of time law with $C=1.16$.

In conclusion, when simulating droplet impact on a liquid film, splashing is observed at high Reynolds numbers and the spreading radius versus time is found to be in good agreement with the power law. Compared with previous MRT multiphase LBMs reaching a maximum Reynolds number of $1000,{ }^{24,35,47}$ our EMRT-MP LBM reaches Reynolds numbers up to 5000 , indicating that the proposed approach is accurate and robust in a simulating complex fluid-fluid interaction at high Reynolds numbers, a high Weber number (220), and at large density ratios (here up to 223). By comparing the computational time of LBGK with our model at the Reynolds numbers of 100 and 1000, we see that our EMRT-MP model requires $11.7 \%$ more time than $\mathrm{LBGK}$, while the last case with a Reynolds number of 5000 cannot be simulated by LBGK.

\section{Droplets on flat solid surface}

\section{Equilibrium contact angles}

The equilibrium contact angle of a droplet deposited on a flat horizontal solid surface is simulated with EMRT-MP LBM while the relative wettability is varied from -1 to 1 . The computational domain is $201 \times 101$ lattices $^{2}$ with a droplet of radius 30 lattices deposited on the flat surface. For boundary conditions, the left and right sides are considered periodic, while the top and bottom surfaces are considered as solid walls. The liquid and vapor densities are $\rho_{l}=7.82$ and $\rho_{v}=0.035$ at a temperature ratio $T / T_{c}=0.74$. The contact angles are measured using the LB-ADSA (low-bond axisymmetric drop shape analysis) plug-in module in ImageJ. ${ }^{49}$

The contact angle of the droplet at equilibrium is determined by the surface wettability and can be approximately

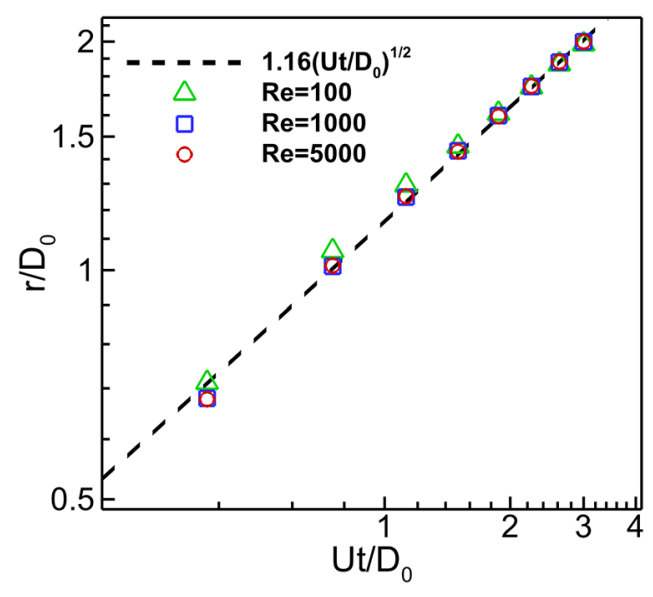

FIG. 8. Spreading radius in functions of non-dimensional time. Symbols: EMRT-MP LBM simulations with different Reynolds numbers (100, 1000, and 5000); dashed line: square root of time law in Ref. 45. 
calculated by Young's equation

$$
\cos (\theta)=\frac{\sigma_{s v}-\sigma_{s l}}{\sigma_{l v}}=\frac{\mathbf{F}_{w}}{\mathbf{F}_{c}},
$$

where $\sigma_{s l}\left(\sigma_{s v}\right)$ is the surface tension between solid and liquid (vapor) and $\sigma_{l v}$ is the surface tension between liquid and vapor. $\mathbf{F}_{c}$ and $\mathbf{F}_{w}$ are the adhesive and cohesive forces described above in Eqs. (17) and (19), respectively. The results obtained by EMRT-MP LBM are shown in Fig. 9. We observe a linear relation between relative wettability and contact angle, which agrees with other simulation results in Refs. 50 and 51.

\section{Droplet impact on flat surfaces}

To evaluate the model in terms of its capacity to resolve dynamic fluid-solid interfacial problems, droplet impacts on weakly hydrophilic and superhydrophobic surfaces are simulated and compared with experimental results. We use a temperature ratio of $T / T_{c}=0.74$, and densities of liquid and vapor are $\rho_{l}=7.78$ and $\rho_{v}=0.038$, respectively. The small change of density ratio is due to a change in surface tension, as explained in Sec. II B 2.

A droplet impact on a surface with a liquid equilibrium contact angle of $89^{\circ}$ is studied in Ref. 52, where the droplet diameter is $2.28 \mathrm{~mm}$ and the impact velocity is $1.0 \mathrm{~m} / \mathrm{s}$. The Reynolds number and Weber number are calculated as $R e=2280$ and $W e=31.5$. In our simulation, the Weber number is the same as the one of the experiment. As described in Ref. 52, at this higher Reynolds number, simulation of the impact process requires a dynamic contact angle in a simplified energy balance approach. Following an approach applied in Ref. 29, we use the same Weber number as in the experiment and set the Reynolds number as $R e=228$. The computational domain size is $600 \times 600 \times 480$ lattices $^{3}$. The initial droplet diameter is $D_{0}=200$ lattices. Figure 10 (Multimedia view) shows snapshots of the droplet shape after impact for both experimental and EMRT-MP LBM simulation results. Physical time in simulation is obtained by taking non-dimensional time $t^{*}=U t / D_{0}$, the same as in experiments. We observe that EMRT-MP LBM reproduces accurately the droplet shapes

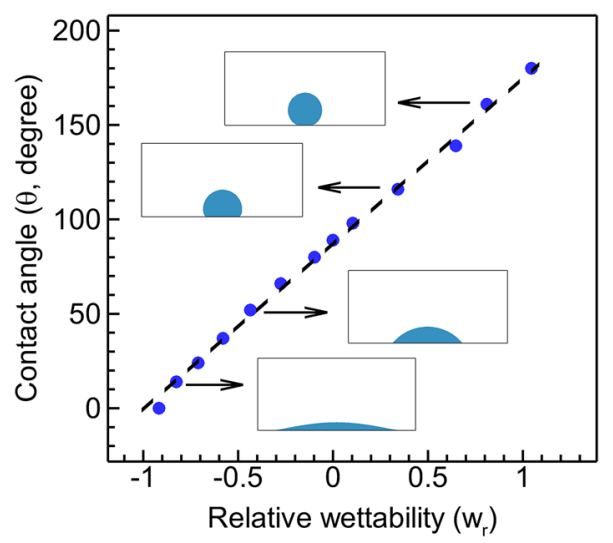

FIG. 9. Equilibrium contact angles for a droplet deposited on a flat solid surface with different relative wettability. Circles: EMRT-LBM results; dashed line: linear fit; insets: simulated configurations.

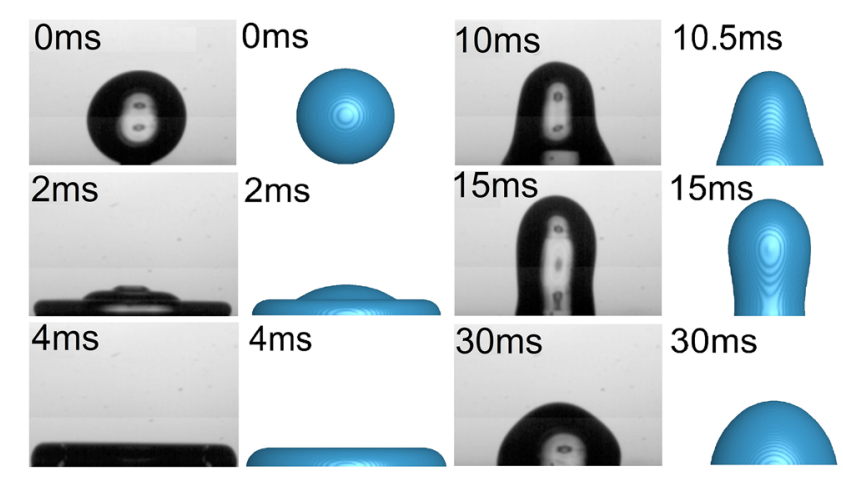

FIG. 10. Snapshots of droplet impact on a hydrophilic surface (contact angle of $89^{\circ}$ ). Left, experiment with initial droplet size $2.28 \mathrm{~mm}$ and impact velocity of $1.0 \mathrm{~m} / \mathrm{s}(\mathrm{Re}=2280$ and $\mathrm{We}=31.5)$ from Ref. 52. [Reprinted with permission from Yokoi et al., "Numerical studies of the influence of the dynamic contact angle on a droplet impacting on a dry surface," Phys. Fluids 21(7), 0721021 (2009). Copyright 2009 AIP Publishing LLC.] Right, EMRT-MP LBM simulation with $\mathrm{Re}=228$ and $\mathrm{We}=31.5$. Multimedia view: https://doi.org/10.1063/1.5016965.4

observed in experiment during the entire regime of spreading and recoiling phases.

For the superhydrophobic case reported in Ref. 53, the initial diameter of the water droplet is $2.28 \mathrm{~mm}$, the impact velocity is $0.35 \mathrm{~m} / \mathrm{s}$, and the contact angle is $180^{\circ}$. The Reynolds and Weber numbers are calculated as $R e=798$ and $W e=3.8$, respectively. In the simulation, the three-dimensional computation domain is $240 \times 240 \times 200$ lattices $^{3}$, the initial droplet diameter is $D_{0}=100$ lattices, and the Reynolds and Weber numbers are the same as in experiment. The snapshots from simulations are compared with the experimental results in Fig. 11 (Multimedia view). The droplet reaches its maximum spreading at $4.675 \mathrm{~ms}$, after which the droplet recedes and departs from the surface. It is clear that the simulation captures all the dynamic processes of droplet impact accurately.

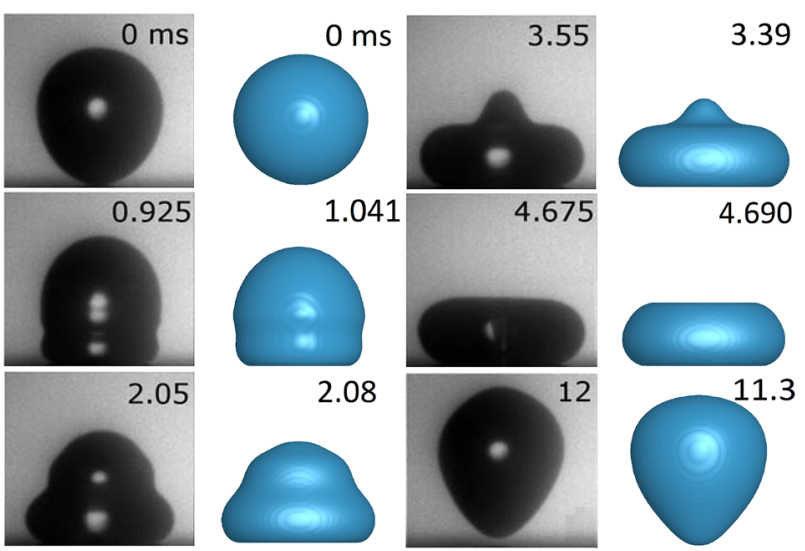

FIG. 11. Snapshots of droplet impact on a superhydrophobic surface (contact angle of $180^{\circ}$ ). Left, experiment of water droplet with initial size $2.28 \mathrm{~mm}$ and an impact velocity of $0.35 \mathrm{~m} / \mathrm{s}(\mathrm{Re}=798$ and $\mathrm{We}=3.8)$ from Ref. 53 . [Reprinted with permission from Vadillo et al., "Dynamic contact angle effects onto the maximum drop impact spreading on solid surfaces," Phys. Fluids 21(12), 122002 (2009). Copyright 2009 AIP Publishing LLC.] Right, EMRT-MP LBM simulation with $\mathrm{Re}=798$ and $\mathrm{We}=3.8$. Multimedia view: https://doi.org/10.1063/1.5016965.5 


\section{Maximum spreading}

The maximum spreading of a droplet impacting a surface has generated great interest in experimental research. ${ }^{54-57}$ Here we study the maximum spreading of a droplet impacting on a superhydrophobic surface for different Reynolds and Weber numbers. We compare the simulation results with an empirical relation.

We use a temperature ratio $T / T_{c}=0.74$ and densities $\rho_{l}=7.78$ and $\rho_{v}=0.038$ for liquid and vapor, respectively. In the simulation, the three-dimensional computation domain contains $660 \times 660 \times 300$ lattices $^{3}$ and the initial droplet diameter is $D_{0}=200$ lattices. The Weber number ranges from 5 to 100 , while the Ohnesorge number (Oh) remains the same in all simulations, $O h=\sqrt{W e} / \operatorname{Re}=0.02$.

For the case of $W e=100$, the droplet evolution after impact is shown in Fig. 12 (Multimedia view). The snapshots record the spreading process until the beginning of the receding phase. In Fig. 13, droplet profiles at different times are given. Toward reaching maximum spreading at $t^{*}=1.813$, the droplet expands and the thickness of lamella continuously decreases while the rim becomes thicker.

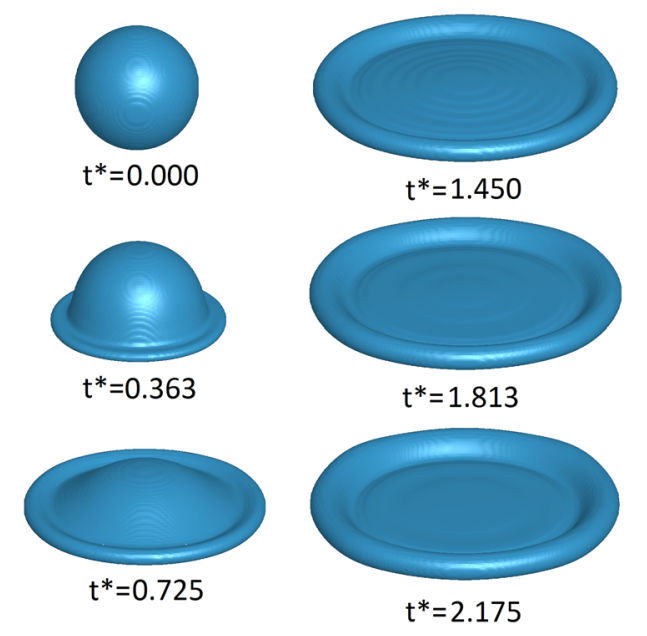

FIG. 12. Snapshots of droplet impact on a superhydrophobic surface (contact angle of $170^{\circ}$ ) with $\mathrm{We}=100$ and $\mathrm{Oh}=0.02$. Multimedia view: https://doi.org/10.1063/1.5016965.6

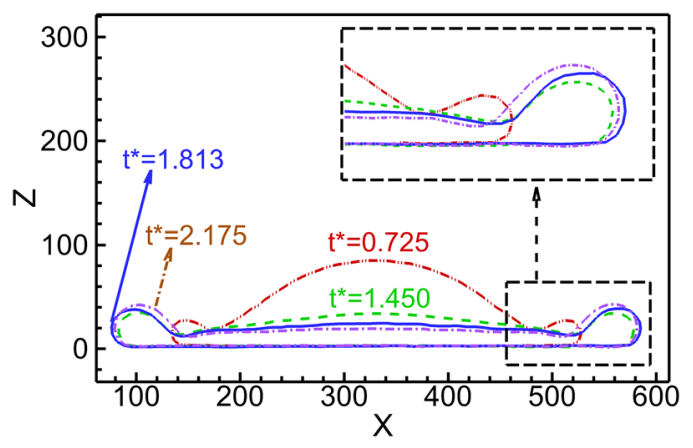

FIG. 13. Profiles of a droplet during impact on a superhydrophobic surface (contact angle of $170^{\circ}$ ) with $\mathrm{We}=100$ and $\mathrm{Oh}=0.02$ at four non-dimensional time instants.
From experimental investigations aiming at finding a universal relation between droplet maximum spreading and Weber/Reynolds number, e.g., Refs. 54 and 55, we retain the following work where the maximum spreading of droplet impact on different surfaces with various liquids is studied in a wide range of impact velocities, with special attention to low impact velocities. ${ }^{57} \mathrm{~A}$ universal relation between rescaled maximum spreading and Weber number is proposed as

$$
\left(\beta_{\max }^{2}-\beta_{u=0}^{2}\right)^{1 / 2} \operatorname{Re}^{-1 / 5}=\frac{W e^{1 / 2}}{A+W e^{1 / 2}},
$$

where $\beta_{\max }=D_{\max } / D_{0}$ is the maximum spreading ratio, $\beta_{u=0}$ is the spreading ratio limit approaching zero impact velocity, and $A=7.6$ is found to be an empirical constant. $\beta_{u=0}=1.226$ is determined by extrapolating the spreading ratio to non-zero velocity droplet impact, as shown in Fig. 14 using the results of maximum spreading ratio as a function of Weber number. Following Ref. 57, the rescaled maximum spreading ratio as a function of Weber number is plotted in Fig. 15. Within the We range explored, the qualitative trend of the universal scaling function is well captured. At higher We around 100, there is a small deviation from the universal scaling curve. To come to a more concise comparison, we have to increase the We, which is subject to future work (simulating higher We with the current model and setup is not yet feasible). Overall, our simulation results agree reasonably well with the universal scaling curve in the simulated We range.

In conclusion, we found good agreement between experiments and our simulations for droplets impacting both hydrophilic and superhydrophobic surfaces. The maximum spreading of a droplet impacting on a superhydrophobic surface is studied, and results show that the rescaled maximum

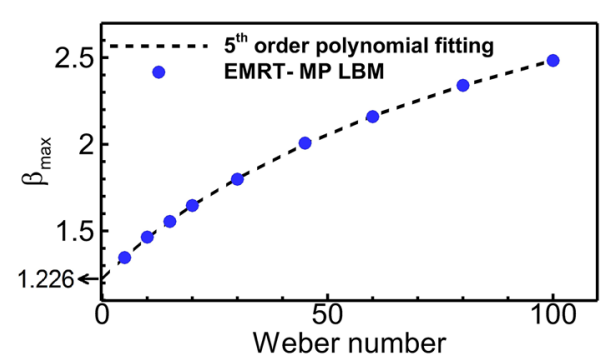

FIG. 14. Maximum spreading ratio as a function of Weber number. A 5th order polynomial fit has been used to obtain the maximum spreading ratio at zero velocity.

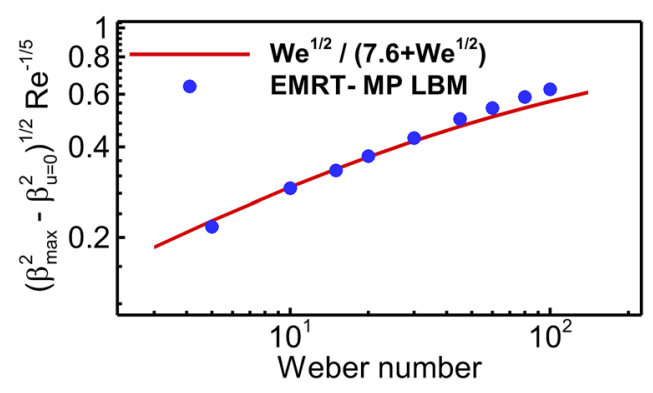

FIG. 15. Rescaled maximum spreading ratio as a function of Weber number for droplet impact on the superhydrophobic surface. Circles: EMRT-MP LBM simulations; solid line: universal scaling law from Ref. 57. 
spreading ratios agree well with a universal scaling law in Ref. 57. All these simulation results show the capacity of our EMRT-MP LBM to capture the complex fluid-solid interactions within a wide range of Reynolds and Weber numbers and at a high density ratio.

\section{CONCLUSION}

In this paper, we couple an entropic multiple-relaxationtime (EMRT) lattice Boltzmann model with a multirange pseudopotential (MP) model for two-phase flow simulations. While the EMRT model makes possible simulating two-phase flow at low fluid viscosity and high density ratio by maximizing the entropy, the MP model adds the major advantage of enabling the tuning of the surface tension. Coupling these two models, the EMRT-MP LBM is capable of simulating fluid flow for a wide range of kinematic viscosity and surface tension values at a high density ratio, in two- and three-dimensional applications.

Droplet splashing on a liquid film with different Reynolds numbers is studied. The spreading radius shows excellent agreement with the empirical equation independent of the Reynolds number. Compared with previous MRT multiphase LBMs which reach an upper bound of the Reynolds number of $1000,{ }^{24,35,47}$ our EMRT-MP LBM is stable and accurate with the Reynolds number up to 5000. A droplet impact on a flat surface with two different wettability conditions is simulated and the spreading and recoiling phases observed in experiments are well captured. Finally, the maximum spreading ratio of a droplet impacting superhydrophobic flat surfaces within a wide range of Weber numbers is studied, and good agreement with the experimental result is found. Compared with previous MRT pseudopotential multiphase LBMs handling the highest Reynolds number of 223 and Weber number of $36.5,{ }^{23}$ our EMRT-MP LBM is stable and accurate for the Reynolds number of 800 and the Weber number of 100. Compared with the ELBM multiphase model, ${ }^{29}$ our EMRT-MP LBM reaches a similar range of Reynolds and Weber numbers, while our density ratio is twice as high when applied to complex droplet dynamics problems, namely, 223 versus 110.

Overall, our EMRT-MP LBM has a better capability to deal with higher Re and We numbers and higher density ratios than previously developed MRT multiphase LBMs. Results in this paper demonstrate that the EMRT-MP LBM is stable and accurate in simulating complex fluid-fluid and fluid-solid interfacial problems such as a droplet splashing on a liquid film and a droplet impacting on a flat surface. The proposed model is shown to be a powerful numerical tool for simulating and analyzing complex two-phase flow dynamics and has the potential to reveal new underlying physics of fluids.

\section{SUPPLEMENTARY MATERIAL}

See supplementary material for the functions of equilibrium distributions and the shear part of the distributions.

\section{ACKNOWLEDGMENTS}

Swiss National Science Foundation (SNF, Project No. 160189) is acknowledged for the financial support. LANL's
Institutional Computing Program is acknowledged for providing the computing support.

${ }^{1} \mathrm{H}$. Li, Y. Zhao, and X. Yuan, "Facile preparation of superhydrophobic coating by spraying a fluorinated acrylic random copolymer micelle solution," Soft Matter 9(4), 1005-1009 (2013).

${ }^{2}$ D. A. Willis and V. Grosu, "Microdroplet deposition by laser-induced forward transfer," Appl. Phys. Lett. 86(24), 244103 (2005).

${ }^{3}$ C. K. Aidun and J. R. Clausen, "Lattice-Boltzmann method for complex flows," Annu. Rev. Fluid Mech. 42(1), 439-472 (2010).

${ }^{4}$ M. C. Sukop and J. D. T. Thorne, Lattice Boltzmann Modeling: An Introduction for Geoscientists and Engineers (Springer, 2010).

${ }^{5}$ Y. Gan, A. Xu, G. Zhang, P. Zhang, and Y. Li, "Lattice Boltzmann study of thermal phase separation: Effects of heat conduction, viscosity and Prandtl number," Europhys. Lett. 97(4), 44002 (2012).

${ }^{6}$ C. W. Hirt and B. D. Nichols, "Volume of fluid (VOF) method for the dynamics of free boundaries," J. Comput. Phys. 39(1), 201-225 (1981).

${ }^{7}$ T. W. H. Sheu, C. H. Yu, and P. H. Chiu, "Development of a dispersively accurate conservative level set scheme for capturing interface in two-phase flows," J. Comput. Phys. 228(3), 661-686 (2009).

${ }^{8}$ M. Sussman, P. Smereka, and S. Osher, "A level set approach for computing solutions to incompressible two-phase flow," J. Comput. Phys. 114(1), 146-159 (1994).

${ }^{9}$ S. Osher and R. P. Fedkiw, "Level set methods: An overview and some recent results," J. Comput. Phys. 169(2), 463-502 (2001).

${ }^{10} \mathrm{X}$. Shan, "Pressure tensor calculation in a class of nonideal gas lattice Boltzmann models," Phys. Rev. E 77(6), 066702 (2008).

${ }^{11} \mathrm{X}$. Shan and H. Chen, "Simulation of nonideal gases and liquid-gas phase transitions by the lattice Boltzmann equation," Phys. Rev. E 49(4), 2941-2948 (1994).

${ }^{12}$ M. Swift, E. Orlandini, W. Osborn, and J. Yeomans, "Lattice Boltzmann simulations of liquid-gas and binary fluid systems," Phys. Rev. E 54(5), 5041-5052 (1996).

${ }^{13}$ A. K. Gunstensen, D. H. Rothman, S. S. Zaleski, and G. Zanetti, "Lattice Boltzmann model of immiscible fluids," Phys. Rev. A 43(8), 4320-4327 (1991).

${ }^{14}$ R. Zheng, H. Liu, J. Sun, and Y. Ba, "Droplet hysteresis investigation on non-wetting striped textured surfaces: A lattice Boltzmann study," Phys. A 411, 53-62 (2014).

${ }^{15}$ T. Reis and T. N. Phillips, "Lattice Boltzmann model for simulating immiscible two-phase flows," J. Phys. A: Math. Theor. 40(14), 4033-4053 (2007).

${ }^{16}$ L. Chen, Q. Kang, Y. Mu, Y. L. He, and W. Q. Tao, "A critical review of the pseudopotential multiphase lattice Boltzmann model: Methods and applications," Int. J. Heat Mass Transfer 76, 210-236 (2014).

${ }^{17}$ Q. Li, K. H. Luo, Q. J. Kang, Y. L. He, Q. Chen, and Q. Liu, "Lattice Boltzmann methods for multiphase flow and phase-change heat transfer," Prog. Energy Combust. Sci. 52, 62-105 (2016).

${ }^{18} \mathrm{X}$. Shan, "Analysis and reduction of the spurious current in a class of multiphase lattice Boltzmann models," Phys. Rev. E 73(4), 047701 (2006).

${ }^{19}$ M. Sbragaglia, R. Benzi, L. Biferale, S. Succi, K. Sugiyama, and F. Toschi, "Generalized lattice Boltzmann method with multirange pseudopotential," Phys. Rev. E 75(2), 026702-1-026702-13 (2007).

${ }^{20}$ S. Chen and G. D. Doolen, "Lattice Boltzmann method for fluid flows," Annu. Rev. Fluid Mech. 30(1), 329-364 (1998)

${ }^{21}$ Y. H. Qian, D. D'Humières, and P. Lallemand, "Lattice BGK models for Navier-Stokes equation," Europhys. Lett. 17(6), 479-484 (1992).

${ }^{22}$ P. Lallemand and L. Luo, "Theory of the lattice Boltzmann method: Dispersion, dissipation, isotropy, Galilean invariance, and stability," Phys. Rev. E 61(6), 6546-6562 (2000).

${ }^{23}$ D. Zhang, K. Papadikis, and S. Gu, "Three-dimensional multi-relaxation time lattice-Boltzmann model for the drop impact on a dry surface at large density ratio," Int. J. Multiphase Flow 64, 11-18 (2014).

${ }^{24}$ Y. Ba, H. Liu, Q. Li, Q. Kang, and J. Sun, "Multiple-relaxation-time colorgradient lattice Boltzmann model for simulating two-phase flows with high density ratio," Phys. Rev. E 94(2), 023310 (2016).

${ }^{25}$ I. V. Karlin, A. N. Gorban, S. Succi, and V. Boffi, "Maximum entropy principle for lattice kinetic equations," Phys. Rev. Lett. 81(1), 6-9 (1998).

${ }^{26}$ I. V. Karlin, A. Ferrante, and H. C. Öttinger, "Perfect entropy functions of the lattice Boltzmann method," Europhys. Lett. 47(2), 182-188 (1999).

${ }^{27}$ S. Ansumali and I. V. Karlin, "Stabilization of the lattice Boltzmann method by the H theorem: A numerical test," Phys. Rev. E 62 (6), 7999-8003 (2000). 
${ }^{28}$ A. Mazloomi M., S. S. Chikatamarla, and I. V. Karlin, "Entropic lattice Boltzmann method for multiphase flows," Phys. Rev. Lett. 114(17), 174502 (2015).

${ }^{29}$ A. Mazloomi M., S. S. Chikatamarla, and I. V. Karlin, "Entropic lattice Boltzmann method for multiphase flows: Fluid-solid interfaces," Phys. Rev. E 92(2), 023308 (2015).

${ }^{30}$ A. Mazloomi M., "Entropic lattice Boltzmann method for two-phase flows," Ph.D. thesis, ETH No. 23905, ETH Zürich, 2016.

${ }^{31}$ I. V. Karlin, F. Bösch, and S. S. Chikatamarla, "Gibbs' principle for the lattice-kinetic theory of fluid dynamics,” Phys. Rev. E 90(3), 031302 (2014).

${ }^{32}$ F. Bösch, S. S. Chikatamarla, and I. V. Karlin, "Entropic multirelaxation lattice Boltzmann models for turbulent flows," Phys. Rev. E 92(4), 043309 (2015).

${ }^{33}$ X. He, S. Chen, and R. Zhang, "A lattice Boltzmann scheme for incompressible multiphase flow and its application in simulation of Rayleigh-Taylor instability," J. Comput. Phys. 663(2), 642-663 (1999).

${ }^{34}$ P. Yuan and L. Schaefer, "Equations of state in a lattice Boltzmann model," Phys. Fluids 18(4), 042101 (2006).

${ }^{35}$ Q. Li, K. H. Luo, and X. J. Li, "Lattice Boltzmann modeling of multiphase flows at large density ratio with an improved pseudopotential model," Phys. Rev. E 87(5), 053301 (2013).

${ }^{36}$ Q. Li, K. H. Luo, Q. J. Kang, and Q. Chen, "Contact angles in the pseudopotential lattice Boltzmann modeling of wetting," Phys. Rev. E 90(5), 053301 (2014).

${ }^{37}$ A. L. Kupershtokh, D. A. Medvedev, and D. I. Karpov, "On equations of state in a lattice Boltzmann method," Comput. Math. Appl. 58(5), 965-974 (2009).

${ }^{38}$ A.-B. Wang and C.-C. Chen, "Splashing impact of a single drop onto very thin liquid films," Phys. Fluids 12(9), 2155-2158 (2000).

${ }^{39}$ C. Mundo, M. Sommerfeld, and C. Tropea, "Droplet-wall collisions: Experimental studies of the deformation and breakup process," Int. J. Multiphase Flow 21(2), 151-173 (1995).

${ }^{40}$ R. L. Vander Wal, G. M. Berger, and S. D. Mozes, "The splash/non-splash boundary upon a dry surface and thin fluid film," Exp. Fluids 40(1), 53-59 (2006).

${ }^{41}$ G. E. Cossali, M. Marengo, A. Coghe, and S. Zhdanov, "The role of time in single drop splash on thin film," Exp. Fluids 36(6), 888-900 (2004).

${ }^{42}$ A. L. Yarin, "DROP IMPACT DYNAMICS: Splashing, spreading, receding, bouncing. ..," Annu. Rev. Fluid Mech. 38(1), 159-192 (2006).
${ }^{43}$ Y. Guo and Y. Lian, "High-speed oblique drop impact on thin liquid films," Phys. Fluids 29(8), 082108 (2017).

${ }^{44}$ S. Asadi and H. Panahi, "A numerical study of a droplet impinging on a liquid surface,” Int. J. Math. Comput. Phys. Electr. Comput. Eng. 5(7), 613-617 (2011).

${ }^{45}$ C. Josserand and S. Zaleski, "Droplet splashing on a thin liquid film," Phys. Fluids 15(6), 1650-1657 (2003).

${ }^{46}$ G. Coppola, G. Rocco, and L. de Luca, "Insights on the impact of a plane drop on a thin liquid film," Phys. Fluids 23(2), 022105 (2011).

${ }^{47} \mathrm{Q}$. Li and K. H. Luo, "Achieving tunable surface tension in the pseudopotential lattice Boltzmann modeling of multiphase flows," Phys. Rev. E 88(5), 053307 (2013).

${ }^{48}$ E. Aslan, I. Taymaz, and A. C. Benim, "Investigation of the lattice Boltzmann SRT and MRT stability for lid driven cavity flow," Int. J. Mater. Mech. Manuf. 2(4), 317-324 (2014).

${ }^{49}$ A. F. Stalder, T. Melchior, M. Müller, D. Sage, T. Blu, and M. Unser, "Lowbond axisymmetric drop shape analysis for surface tension and contact angle measurements of sessile drops," Colloids Surf., A 364(1-3), 72-81 (2010).

${ }^{50}$ L. Chen, Q. Kang, B. A. Robinson, Y. L. He, and W. Q. Tao, "Porescale modeling of multiphase reactive transport with phase transitions and dissolution-precipitation processes in closed systems," Phys. Rev. E 87(4), 043306 (2013).

${ }^{51} \mathrm{P}$. Yuan, "Thermal lattice Boltzmann two-phase flow model for fluid dynamics," Ph.D. dissertation (University of Pittsburgh, 2005).

${ }^{52}$ K. Yokoi, D. Vadillo, J. Hinch, and I. Hutchings, "Numerical studies of the influence of the dynamic contact angle on a droplet impacting on a dry surface," Phys. Fluids 21(7), 072102 (2009).

${ }^{53}$ D. C. Vadillo, A. Soucemarianadin, C. Delattre, and D. C. D. Roux, "Dynamic contact angle effects onto the maximum drop impact spreading on solid surfaces," Phys. Fluids 21(12), 122002 (2009).

${ }^{54}$ D. Richard, D. Quéré, C. Clanet, and C. Béguin, "Maximal deformation of an impacting drop," J. Fluid Mech. 517, 199-208 (2004).

${ }^{55}$ N. Laan, K. G. De Bruin, D. Bartolo, C. Josserand, and D. Bonn, "Maximum diameter of impacting liquid droplets," Phys. Rev. Appl. 2(4), 044018 (2014).

${ }^{56}$ G. Lagubeau, M. A. Fontelos, C. Josserand, A. Maurel, V. Pagneux, and P. Petitjeans, "Spreading dynamics of drop impacts," J. Fluid Mech. 713, 50-60 (2012).

${ }^{57}$ J. B. Lee, N. Laan, K. G. de Bruin, G. Skantzaris, N. Shahidzadeh, D. Derome, J. Carmeliet, and D. Bonn, "Universal rescaling of drop impact on smooth and rough surfaces," J. Fluid Mech. 786, R4 (2016). 